\title{
Mechanically deposited tungsten disulfide saturable absorber for low-threshold Q-switched erbium-doped fiber laser
}

\begin{abstract}
In this paper, we report a Q-switched erbium-doped fiber laser incorporating tungsten disulfide $\left(\mathrm{WS}_{2}\right)$ as the saturable absorber. A direct mechanical deposition technique using a scotch tape is employed to place the $\mathrm{WS}_{2}$ powder onto the tip of a fiber ferrule. Several runs of mechanical extraction are performed to reduce the thickness of $\mathrm{WS}_{2}$ powder on the fiber tip. The fabricated $\mathrm{WS}_{2}$ saturable absorber exhibits a saturation intensity of $548.6 \mathrm{MW} / \mathrm{cm}^{2}$, modulation depth of $4.1 \%$ and non-saturable loss of $67.8 \%$. A ring cavity erbium-doped fiber laser integrating the $\mathrm{WS}_{2}$ saturable absorber yields continuous wave lasing and Q-switching threshold at pump power of 10 and $12.7 \mathrm{~mW}$, respectively. This Q-switching pump power threshold is the lowest to the best of the authors' knowledge. The proposed technique of direct mechanical deposition is inexpensive, significantly faster and simpler compared to previously reported methods.
\end{abstract}

Keyword: Tungsten disulfide; Saturable absorber; Q-switched; Erbium-doped fiber laser 